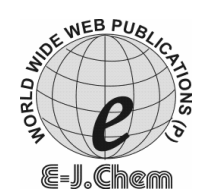

http://www.e-journals.net
ISSN: 0973-4945; CODEN ECJHAO

E-Journal of Chemistry

2008, 5(S2), 1154-1158

\title{
Synthesis and Spectral Study of Novel Norfloxacin Derivatives
}

\author{
PRADEEP YADAV and Y.C.JOSHI* \\ Department of Chemistry, \\ University of Rajasthan, \\ Jaipur- 302004-Rajasthan, India \\ pradeepyadav78@gmail.com
}

Received 5 October 2007; Accepted 3 December 2007

\begin{abstract}
Reaction of [1-ethyl-6-fluoro-1,4-dihydro-4-oxo-7-(1-piperazinyl)quinolone-3-carboxylic acid (norfloxacin) with thiazole / benzothiazole diazonium chloride to get new piperazine substituted norfloxacin derivative. These norfloxacin derivatives were further condensed with various $\beta$-diketone to get novel acid derivatives of 1-Ethyl-6-fluoro-4-oxo-7- [4 (thiazol-2yldiazenyl)-piperzin-1-yl]-1,4-dihydro-quinoline-3-carboxylic acid (6a-e) and 7-(4-(benzo[d]thiazol-2-yldiazenyl)piperazin-1-yl)-1-ethyl-6-fluoro-4-oxo-1, 4dihydroquinoline-3-carboxylic acid ( $6 \mathrm{f}-\mathrm{j})$. Structures of these compounds were established on the basis of spectral studies viz. IR, ${ }^{1} \mathrm{H}$ NMR etc.
\end{abstract}

Keyword: Norfloxacin, $\beta$-Diketone, Thiazole, Benzothiazole

\section{Introduction}

The earliest antibacterial agents to be used clinically in the 1920 were the synthetic sulfonamides. Since then, quinolones were the only synthetic agents that play a major role in the treatment of bacterial community or hospital-acquired diseases. The excellent pharmacokinetic properties, high antimicrobial activity, and few side effects that most quinolones demonstrate explain their widespread use in clinical practice ${ }^{1}$ The compounds have had good success against gram-negative bacteria, but resistance of Gram-positive pathogens, such as Staphylococcus aureus, has become a problem ${ }^{2-8}$. In addition, certain adverse events (e.g, CNS side effects, phototoxicity, and arthropathy) became apparent, although the more serious events are rare ${ }^{9-10}$. Thus, despite many advances in the fluoroquinolone field, there exists a continuous need for novel quinolones to overcome the limitations of existing drugs. 
The 1,4-dihydro-4-oxopyridine-3-carboxylic acid associated with a 5,6-fused aromatic ring is the common chemical feature of bactericidal quinolones. In the resulting bicyclic ring, the 1,5,6,7, and 8-positions are the major targets of chemical variation but, synthetic efforts for improved potency have been the main focus on 7-position ${ }^{11-12}$. Both activity spectrum and kinetic profile can be controlled at C-7. The most common substituents are cyclic amino groups, for example, piperazine or pyrrolidine rings; other groups have been less successful. Piperazine rings are particularly common (e.g. ciprofloxacin, norfloxacin or enoxacin) and confer potency against gram-negative bacteria ${ }^{11-12}$. The first generation of 7-piperazinyl quinolones, exemplified by ciprofloxacin, norfloxacin, and enoxacin, have high activity against Gram-negative species and atypicals, but moderate activity against Gram-positive bacteria ${ }^{2}$. In this paper we report the synthesis and the spectral study of novel $\beta$-dicarbonyl derivative of [1-ethyl-6-fluoro-1,4-dihydro-4-oxo-7-(1-piperazinyl)-quinolone3 -carboxylic acid (norfloxacin).

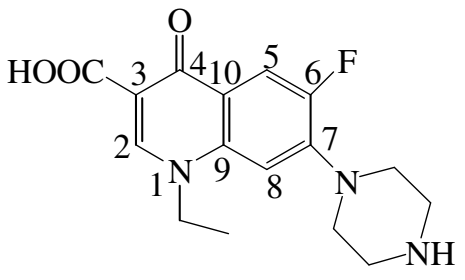

\section{Experimental}

Structure of norfloxacin

All the melting points are uncorrected (Table 1). Using Perkin-Elmer CHNS/O analyzer 2400 carried out the elemental analysis. Infrared spectra were recorded in $\mathrm{KBr}(\mathrm{AR})$ pellets on a Nicolet-Megna-FT-IR 550 spectrometer. ${ }^{1} \mathrm{H}$ NMR spectra were recorded on model DRX 300 at $300.13 \mathrm{MHz}$ in DMSO-D6 solvent; using TMS as internal standard ${ }^{1} \mathrm{H}$ NMR spectra were recorded at room temperature and $2 \mathrm{mg} / \mathrm{mL}$. The purity of compounds was tested by pre coated silica gel 60F254 TLC plates from E. Merck in different solvent systems.

\section{General method of preparation of diazo derivatives (3a-b)}

A mixture of norfloxacin $(1.655 \mathrm{~g}, 005 \mathrm{~mol})$ and sodium bicarbonate $(1.0 \mathrm{~g}$, excess $)$ in 10 $\mathrm{mL}$ of acetonitrile was stirred at $50^{\circ} \mathrm{C}$ for 4 hour. Reaction mixture was cooled to $0^{\circ} \mathrm{C}$ and diazonium chloride of 2-aminothiazole $(0.5 \mathrm{~g}, 0.005 \mathrm{~mol}) / 2$-aminobenzothiazole $(0.75 \mathrm{~g}$, $0.005 \mathrm{~mol})(2 \mathrm{a}-\mathrm{b})$ was added. The mixture was stirred at magnetic stirrer for 5 hours at 0 $5^{0} \mathrm{C}$.Acetonitrile was removed under reduced pressure and the solid residue was partitioned between chloroform/water. The organic layer was dried by sodium sulphate, recrystallized in hexane acetone mixture. The purity of compound was checked by TLC in chloroform: acetone: 50:50 mixture.

General method for preparation of 1-ethyl-6-fluoro-4-oxo-7-(4-(thiazol-2-yldiazenyl) piperazin -1-yl)-1,4-dihydroquinoline-3-carboxylic acid derivatives (6a-j)

Compound (3a-b) was dissolved in thionyl chloride and refluxed for 4 hours to get acid chloride derivative (4a-b). Excess thionyl chloride was removed under reduced pressure and sodium salt of $\beta$-diketones (prepared using $\mathrm{NaOMe}$ and $\beta$-diketone (5a-e) under $\mathrm{N}_{2}$ atmosphere) was added and stirred overnight at RT. Reaction mixture was quenched with ice cold water and stirred for $30 \mathrm{~min}$. The precipitate was filtered and washed with acetone to get (6a-j), which were further purified through column chromatography in some cases. 
<smiles>CCn1cc(C(=O)O)c(=O)c2cc(F)c(N3CCNCC3)cc21</smiles>

1

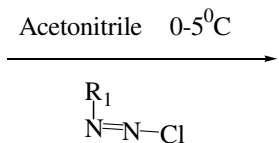

(2a-b)

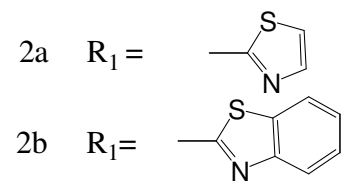

\section{Results and Discussion}

\section{Scheme 1}

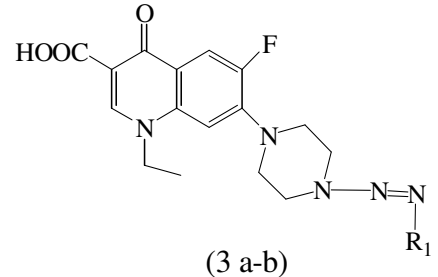

$\mathrm{SOCl}_{2}$

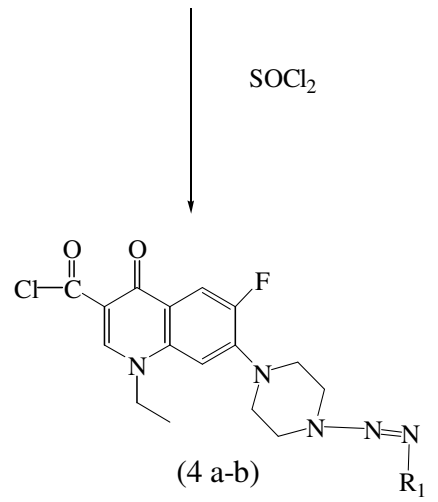

Sodium methoxide $\mid$<smiles>[R]N=NN1CCN(c2cc3c(cc2F)c(=O)c(C(=O)C(C([R])=O)C([R])=O)cn3CC)CC1</smiles>

A series of 1-ethyl-6-fluoro-4-oxo-7-(4-(thiazol-2-yldiazenyl)piperazin -1-yl)-1,4-dihydroquinoline-3-carboxylic acid derivatives (6a-e) and 7-(4-(benzo[d]thiazol-2-yldiazenyl) piperazin-1-yl)-1-ethyl-6-fluoro-4-oxo-1,4-dihydroquinoline-3-carboxylic acid derivatives (6f-j) were synthesized in moderate yields using the synthetic route outlined in Scheme 1. Structure of these synthesized compounds were established on the basis of IR, ${ }^{1} \mathrm{H}$ NMR and elemental analysis. (Table 1\&2). Norfloxacin was treated with thiazole/ benzothiazole diazonium chloride in presence of base to give piperazine substituted norfloxacin derivatives (3a-b). The acid part of these derivatives was converted to acid chloride using thionyl chloride which was further condensed with various $\beta$-diketone (5a-e) to get (6a-j). 
Table 1. Substitution pattern and physical properties for novel norfloxacin derivatives (3a-b, 6a-j)

\begin{tabular}{|c|c|c|c|c|c|c|c|c|}
\hline \multirow{2}{*}{$\begin{array}{l}\text { S. } \\
\text { No }\end{array}$} & \multirow{2}{*}{$\mathrm{R}_{2}$} & \multirow{2}{*}{$\mathrm{R}_{3}$} & \multirow{2}{*}{${ }^{\mathrm{m}} \mathrm{C} . \mathrm{C}}$. & \multirow{2}{*}{ Yield } & \multirow{2}{*}{$\begin{array}{l}\text { Molecular } \\
\text { formula }\end{array}$} & \multicolumn{3}{|c|}{ Elemental data Calculated (Found) \% } \\
\hline & & & & & & $\mathrm{C}$ & $\mathrm{H}$ & $\mathrm{N}$ \\
\hline $3 a$ & -- & -- & 142 & 51 & $\mathrm{C}_{19} \mathrm{H}_{19} \mathrm{FN}_{6} \mathrm{O}_{3} \mathrm{~S}$ & 53.01(53.0) & $4.45(4.40)$ & $19.52(19.50)$ \\
\hline $3 b$ & -- & -- & 154 & 56 & $\mathrm{C}_{23} \mathrm{H}_{21} \mathrm{FN}_{6} \mathrm{O}_{3} \mathrm{~S}$ & $57.49(57.47)$ & $4.41(4.40)$ & $17.49(17.47)$ \\
\hline $6 a$ & $\mathrm{CH}_{3}$ & $\mathrm{CH}_{3}$ & 190 & 54 & $\mathrm{C}_{24} \mathrm{H}_{25} \mathrm{FN}_{6} \mathrm{O}_{4} \mathrm{~S}$ & $56.24(56.20)$ & $4.92(4.90)$ & $16.40(16.38)$ \\
\hline $6 b$ & $\mathrm{CH}_{3}$ & $\mathrm{C}_{6} \mathrm{H}_{5}$ & 180 & 52 & $\mathrm{C}_{29} \mathrm{H}_{27} \mathrm{FN}_{6} \mathrm{O}_{4} \mathrm{~S}$ & $60.62(60.60)$ & $4.74(4.72)$ & $14.63(14.62)$ \\
\hline $6 c$ & $\mathrm{C}_{6} \mathrm{H}_{5}$ & $\mathrm{C}_{6} \mathrm{H}_{5}$ & 206 & 53 & $\mathrm{C}_{34} \mathrm{H}_{29} \mathrm{FN}_{6} \mathrm{O}_{4} \mathrm{~S}$ & $64.14(64.13)$ & $4.59(4.58)$ & $13.20(13.19)$ \\
\hline $6 d$ & $\mathrm{CH}_{3}$ & $\mathrm{OC}_{2} \mathrm{H}_{5}$ & 214 & 54 & $\mathrm{C}_{25} \mathrm{H}_{27} \mathrm{FN}_{6} \mathrm{O}_{5} \mathrm{~S}$ & $55.34(55.32)$ & $5.02(5.00)$ & $15.49(15.48)$ \\
\hline $6 e$ & $\mathrm{OC}_{2} \mathrm{H}_{5}$ & $\mathrm{OC}_{2} \mathrm{H}_{5}$ & 154 & 62 & $\mathrm{C}_{26} \mathrm{H}_{29} \mathrm{FN}_{6} \mathrm{O}_{6} \mathrm{~S}$ & $54.54(54.52)$ & $5.10(5.09)$ & $14.68(14.67)$ \\
\hline $6 f$ & $\mathrm{CH}_{3}$ & $\mathrm{CH}_{3}$ & 175 & 58 & $\mathrm{C}_{28} \mathrm{H}_{27} \mathrm{FN}_{6} \mathrm{O}_{4} \mathrm{~S}$ & $59.77(59.76)$ & $4.84(4.83)$ & $14.94(14.92)$ \\
\hline $6 \mathrm{~g}$ & $\mathrm{CH}_{3}$ & $\mathrm{C}_{6} \mathrm{H}_{5}$ & 182 & 54 & $\mathrm{C}_{33} \mathrm{H}_{29} \mathrm{FN}_{6} \mathrm{O}_{4} \mathrm{~S}$ & $63.45(63.44)$ & $4.68(4.67)$ & $13.45(13.44)$ \\
\hline $6 \mathrm{~h}$ & $\mathrm{C}_{6} \mathrm{H}_{5}$ & $\mathrm{C}_{6} \mathrm{H}_{5}$ & 193 & 57 & $\mathrm{C}_{38} \mathrm{H}_{31} \mathrm{FN}_{6} \mathrm{O}_{4} \mathrm{~S}$ & $66.46(66.45)$ & $4.55(4.54)$ & $12.24(12.23)$ \\
\hline $6 \mathrm{i}$ & $\mathrm{CH}_{3}$ & $\mathrm{OC}_{2} \mathrm{H}_{5}$ & 218 & 55 & $\mathrm{C}_{29} \mathrm{H}_{29} \mathrm{FN}_{6} \mathrm{O}_{5} \mathrm{~S}$ & $58.77(58.76)$ & 4.93(4.92) & $14.18(14.17)$ \\
\hline $6 \mathrm{j}$ & $\mathrm{OC}_{2} \mathrm{H}_{5}$ & $\mathrm{OC}_{2} \mathrm{H}_{5}$ & 171 & 58 & $\mathrm{C}_{30} \mathrm{H}_{31} \mathrm{FN}_{6} \mathrm{O}_{6} \mathrm{~S}$ & $57.87(57.85)$ & $5.02(5.00)$ & $13.50(13.49)$ \\
\hline
\end{tabular}

Table 2.

\begin{tabular}{|c|c|c|}
\hline Comp & IR $(\mathrm{KBr}) \mathrm{cm}^{-1}$ & $1 \mathrm{H} \mathrm{NMR}\left(\mathrm{CDCl}_{3} / \mathrm{DMSO} \mathrm{d}_{6}\right) \delta \mathrm{ppm}$ \\
\hline $3 \mathrm{a}$ & $\begin{array}{l}3495,3080,2920,1725,15 \\
81,1428,691\end{array}$ & $\begin{array}{l}1.29\left(3 \mathrm{H}, \mathrm{t}, \mathrm{CH}_{3}\right) 3.4\left(2 \mathrm{H}, \mathrm{q}, \mathrm{CH}_{2}\right) 3.8\left(8 \mathrm{H}, \mathrm{m}, \mathrm{N}-\mathrm{CH}_{2^{-}}\right. \\
\left.\mathrm{CH}_{2} \mathrm{~N}\right), 7.45(1 \mathrm{H}, \mathrm{d}, \mathrm{C} 8-\mathrm{H}) 7.63\left(1 \mathrm{H}, \mathrm{d}, \mathrm{C}_{5}-\mathrm{H}\right), 7.80 \\
\left(1 \mathrm{H}, \mathrm{s}, \mathrm{C}_{2}-\mathrm{H}\right), 7.52(1 \mathrm{H}, \mathrm{d}, \mathrm{CH}) 8.1(1 \mathrm{H}, \mathrm{d}, \mathrm{CH}) .11 .2 \\
(1 \mathrm{H}, \mathrm{s}, \mathrm{COOH})\end{array}$ \\
\hline $3 b$ & $\begin{array}{l}3483,3085, \\
2923,1725,1584,1423,672\end{array}$ & $\begin{array}{l}1.24\left(3 \mathrm{H}, \mathrm{t}, \mathrm{CH}_{3}\right) 3.42\left(2 \mathrm{H}, \mathrm{q}, \mathrm{CH}_{2}\right) 3.9-4.1(8 \mathrm{H}, \mathrm{m}, \mathrm{N}- \\
\left.\mathrm{CH}_{2}-\mathrm{CH}_{2} \mathrm{~N}\right), 7.50\left(1 \mathrm{H}, \mathrm{d}, \mathrm{C}_{8}-\mathrm{H}\right) 7.63\left(1 \mathrm{H}, \mathrm{d}, \mathrm{C}_{5}-\mathrm{H}\right) \\
7.78\left(1 \mathrm{H}, \mathrm{s}, \mathrm{C}_{2}-\mathrm{H}\right), 7.4-7.6(4 \mathrm{H}, \mathrm{m}, \mathrm{Ar}-\mathrm{H}) .11 .2 \\
(1 \mathrm{H}, \mathrm{s}, \mathrm{COOH})\end{array}$ \\
\hline $6 a$ & $\begin{array}{l}3090, \\
2921,1715,1585,1432,684\end{array}$ & 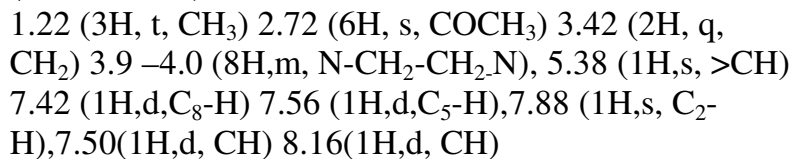 \\
\hline $6 b$ & $\begin{array}{l}3085,2921,1723,1580,14 \\
32,670\end{array}$ & $\begin{array}{l}1.25\left(3 \mathrm{H}, \mathrm{t}, \mathrm{CH}_{3}\right) 2.72\left(3 \mathrm{H}, \mathrm{s}, \mathrm{COCH}_{3}\right) 3.38\left(2 \mathrm{H}, \mathrm{q}, \mathrm{CH}_{2}\right) \text {, } \\
3.9-4.0\left(8 \mathrm{H}, \mathrm{m}, \mathrm{N}-\mathrm{CH}_{2}-\mathrm{CH}_{2} \mathrm{~N}\right), 5.32(1 \mathrm{H}, \mathrm{s},>\mathrm{CH}) 7.38 \\
\left(1 \mathrm{H}, \mathrm{d}, \mathrm{C}_{8}-\mathrm{H}\right) 7.65\left(1 \mathrm{H}, \mathrm{d}, \mathrm{C}_{5}-\mathrm{H}\right), 7.86\left(1 \mathrm{H}, \mathrm{s}, \mathrm{C}_{2}-\mathrm{H}\right), 7.4- \\
7.5(5 \mathrm{H}, \mathrm{m}, \mathrm{Ar}-\mathrm{H}) 7.54(1 \mathrm{H}, \mathrm{d}, \mathrm{CH}) 8.12(1 \mathrm{H}, \mathrm{d}, \mathrm{CH})\end{array}$ \\
\hline $6 c$ & $\begin{array}{l}3081,2922, \\
1721,1591,1423,685\end{array}$ & 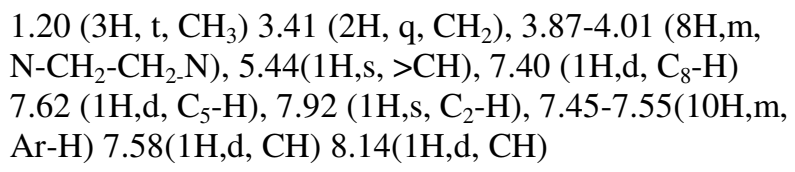 \\
\hline $6 \mathrm{~d}$ & $\begin{array}{l}3080,2914,1719,1578, \\
1424,688\end{array}$ & $\begin{array}{l}1.23\left(3 \mathrm{H}, \mathrm{t}, \mathrm{CH}_{3}\right) 2.13\left(3 \mathrm{H}, \mathrm{t}, \mathrm{CH}_{2}-\mathrm{CH}_{3}\right) 2.71(3 \mathrm{H}, \mathrm{s}, \\
\left.\mathrm{COCH}_{3}\right) 3.42\left(2 \mathrm{H}, \mathrm{q}, \mathrm{CH}_{2}\right), 3.8\left(8 \mathrm{H}, \mathrm{m}, \mathrm{N}-\mathrm{CH}_{2}-\mathrm{CH}_{2} \mathrm{~N}\right), \\
4.0\left(2 \mathrm{H}, \mathrm{q}, \mathrm{O}-\mathrm{CH}_{2}\right) 5.30(1 \mathrm{H}, \mathrm{s},>\mathrm{CH}), 7.47\left(1 \mathrm{H}, \mathrm{d}, \mathrm{C}_{8}-\mathrm{H}\right) \\
7.66\left(1 \mathrm{H}, \mathrm{d}, \mathrm{C}_{5}-\mathrm{H}\right), 7.76\left(1 \mathrm{H}, \mathrm{s}, \mathrm{C}_{2}-\mathrm{H}\right), 7.54(1 \mathrm{H}, \mathrm{d}, \mathrm{CH}) \\
8.11(1 \mathrm{H}, \mathrm{d}, \mathrm{CH})\end{array}$ \\
\hline
\end{tabular}




\begin{tabular}{|c|c|c|}
\hline $6 e$ & $\begin{array}{l}3080,2921,1720,1575 \\
1424,681\end{array}$ & $\begin{array}{l}1.25\left(3 \mathrm{H}, \mathrm{t}, \mathrm{CH}_{3}\right) 2.14\left(6 \mathrm{H}, \mathrm{t}, \mathrm{CH}_{2}-\mathrm{CH}_{3}\right), 3.45\left(2 \mathrm{H}, \mathrm{t}, \mathrm{CH}_{2}\right) \\
3.8-4.0\left(8 \mathrm{H}, \mathrm{m}, \mathrm{N}-\mathrm{CH}_{2}-\mathrm{CH}_{2} \mathrm{~N}\right), 3.9\left(4 \mathrm{H}, \mathrm{q}, \mathrm{O}-\mathrm{CH}_{2}\right) 5.32 \\
(1 \mathrm{H}, \mathrm{s},>\mathrm{CH}), 7.48\left(1 \mathrm{H}, \mathrm{d}, \mathrm{C}_{8}-\mathrm{H}\right) 7.69\left(1 \mathrm{H}, \mathrm{d}, \mathrm{C}_{5}-\mathrm{H}\right), 7.75 \\
\left(1 \mathrm{H}, \mathrm{s}, \mathrm{C}_{2}-\mathrm{H}\right), 7.55(1 \mathrm{H}, \mathrm{d}, \mathrm{CH}) 8.09(1 \mathrm{H}, \mathrm{d}, \mathrm{CH})\end{array}$ \\
\hline $6 f$ & $\begin{array}{l}3094,2918, \\
1714,1580,1418,690\end{array}$ & $\begin{array}{l}1.19\left(3 \mathrm{H}, \mathrm{t}, \mathrm{CH}_{3}\right) 2.72\left(6 \mathrm{H}, \mathrm{s}, \mathrm{COCH}_{3}\right), 3.42(2 \mathrm{H}, \mathrm{q}, \\
\left.\mathrm{CH}_{2}\right) 3.9-4.0\left(8 \mathrm{H}, \mathrm{m}, \mathrm{N}_{-}-\mathrm{CH}_{2}-\mathrm{CH}_{2} \mathrm{~N}\right), 5.33(1 \mathrm{H}, \mathrm{s},>\mathrm{CH} \\
\left.\text { 7.48(1H,d, } \mathrm{C}_{8}-\mathrm{H}\right) 7.65\left(1 \mathrm{H}, \mathrm{d}, \mathrm{C}_{5}-\mathrm{H}\right), 7.82\left(1 \mathrm{H}, \mathrm{s}, \mathrm{C}_{2}-\mathrm{H}\right) \text {, } \\
\text { 7.4-7.6 (4H,m, Ar-H). }\end{array}$ \\
\hline $6 \mathrm{~g}$ & $\begin{array}{l}3080,2920,1713,1581, \\
1420,691\end{array}$ & $\begin{array}{l}1.20\left(3 \mathrm{H}, \mathrm{t}, \mathrm{CH}_{3}\right) 2.72\left(3 \mathrm{H}, \mathrm{s}, \mathrm{COCH}_{3}\right), 3.44(2 \mathrm{H}, \mathrm{q}, \\
\left.\mathrm{CH}_{2}\right) 3.9-4.1\left(8 \mathrm{H}, \mathrm{m}, \mathrm{N}_{-} \mathrm{CH}_{2}-\mathrm{CH}_{2} \mathrm{~N}\right), 5.35(1 \mathrm{H}, \mathrm{s},>\mathrm{CH} \\
\left.\text { 7.49(1H,d, } \mathrm{C}_{8}-\mathrm{H}\right) 7.65\left(1 \mathrm{H}, \mathrm{d}, \mathrm{C}_{5}-\mathrm{H}\right), 7.83\left(1 \mathrm{H}, \mathrm{s}, \mathrm{C}_{2}-\mathrm{H}\right) \text {, } \\
\text { 7.4-7.6(9H,m, Ar-H). }\end{array}$ \\
\hline $6 \mathrm{~h}$ & $\begin{array}{l}3082,2920,1720,1582, \\
1422,692\end{array}$ & $\begin{array}{l}1.24\left(3 \mathrm{H}, \mathrm{t}, \mathrm{CH}_{3}\right), 3.40\left(2 \mathrm{H}, \mathrm{q}, \mathrm{CH}_{2}\right) 3.8-3.9(8 \mathrm{H}, \mathrm{m}, \mathrm{N}- \\
\left.\mathrm{CH}_{2}-\mathrm{CH}_{2} \mathrm{~N}\right), 5.36(1 \mathrm{H}, \mathrm{s},>\mathrm{CH}) 7.48\left(1 \mathrm{H}, \mathrm{d}, \mathrm{C}_{8}-\mathrm{H}\right) 7.65 \\
\left(1 \mathrm{H}, \mathrm{d}, \mathrm{C}_{5}-\mathrm{H}\right), 7.78\left(1 \mathrm{H}, \mathrm{s}, \mathrm{C}_{2}-\mathrm{H}\right), 7.5-7.6(14 \mathrm{H}, \mathrm{m}, \mathrm{Ar}-\mathrm{H}) .\end{array}$ \\
\hline $6 \mathrm{i}$ & $\begin{array}{l}3084,2917,1721,1581, \\
1432,681\end{array}$ & $\begin{array}{l}1.20\left(3 \mathrm{H}, \mathrm{t}, \mathrm{CH}_{3}\right) 2.13\left(3 \mathrm{H}, \mathrm{t}, \mathrm{CH}_{2}-\mathrm{CH}_{3}\right) 2.71(3 \mathrm{H}, \mathrm{s} \\
\left.\mathrm{COCH}_{3}\right), 3.42\left(2 \mathrm{H}, \mathrm{q}, \mathrm{CH}_{2}\right) 3.8-4.1\left(10 \mathrm{H}, \mathrm{m}, \mathrm{N}-\mathrm{CH}_{2^{-}}\right. \\
\left.\mathrm{CH}_{2} \mathrm{~N}, \mathrm{O}-\mathrm{CH}_{2}\right), 5.40(1 \mathrm{H}, \mathrm{s},>\mathrm{CH}) 7.35\left(1 \mathrm{H}, \mathrm{d}, \mathrm{C}_{8}-\mathrm{H}\right) 7.65 \\
\left(1 \mathrm{H}, \mathrm{d}, \mathrm{C}_{5}-\mathrm{H}\right), 7.81\left(1 \mathrm{H}, \mathrm{s}, \mathrm{C}_{2}-\mathrm{H}\right), 7.4-7.6(4 \mathrm{H}, \mathrm{m}, \mathrm{Ar}-\mathrm{H}) .\end{array}$ \\
\hline $6 \mathrm{j}$ & $\begin{array}{l}3080,2922,1719,1578, \\
1420,690\end{array}$ & $\begin{array}{l}1.25\left(3 \mathrm{H}, \mathrm{t}, \mathrm{CH}_{3}\right) 2.12\left(6 \mathrm{H}, \mathrm{t}, \mathrm{CH}_{2}-\mathrm{CH}_{3}\right) 3.45(2 \mathrm{H}, \mathrm{q} \\
\left.\mathrm{CH}_{2}\right), 3.8-4.0\left(12 \mathrm{H}, \mathrm{m}, \mathrm{N}-\mathrm{CH}_{2}-\mathrm{CH}_{2}-\mathrm{N} \& \mathrm{O}_{-} \mathrm{CH}_{2}\right) \\
5.37(1 \mathrm{H}, \mathrm{s},>\mathrm{CH}) 7.47\left(1 \mathrm{H}, \mathrm{d}, \mathrm{C}_{8}-\mathrm{H}\right) 7.62\left(1 \mathrm{H}, \mathrm{d}, \mathrm{C}_{5^{-}}\right. \\
\mathrm{H}), 7.81\left(1 \mathrm{H}, \mathrm{s}, \mathrm{C}_{2}-\mathrm{H}\right), 7.5-7.6(4 \mathrm{H}, \mathrm{m}, \mathrm{Ar}-\mathrm{H}) .\end{array}$ \\
\hline
\end{tabular}

Acknowledgement

Authors are thankful to Head Department of Chemistry, University of Rajasthan, Jaipur for providing necessary laboratory facilities and spectral data's

\section{References}

1. Andriole V T, Drugs, 1999, 58(Suppl. 2), 1.

2. Emami S, Shafiee A and Foroumadi A, Mini-Rev. Med.Chem, 2006, 6, 109.

3. Blumberg H M, RimLand D, Carroll D J, Terry P and Wachsmuth I K, J Infect Dis., 1991, 163, 1279.

4. Peterson L R, Quick J N, Jensen B, Homann S, Johnson S and Tenquist J, Arch Intern Med., 1990, 150, 2151

5. Schaefler S, J Clin Microbiol., 1989, 27, 335.

6. Hooper D C, Lancet Infect Dis., 2002, 2, 530.

7. Bearden D T and Danziger L H, Pharmacotherapy, 2001, 21, 224S.

8. Piddock L J V, Drugs, 1999, 58(Suppl. 2), 11.

9. Lietman P S, Drugs, 1995, 49(Suppl. 2), 159.

10. Ball P, Mandell L, Niki Y and Tillotson G, Drug Safety, 1999, 21, 407.

11. Domagala J M, J Antimicrob Chemother., 1994, 33, 685.

12. Asahina Y, Ishizaki T and Suzue S, Prog Drug Res., 1992, 38, 57. 


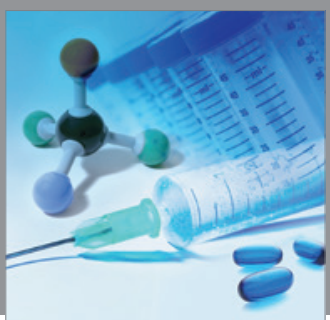

International Journal of

Medicinal Chemistry

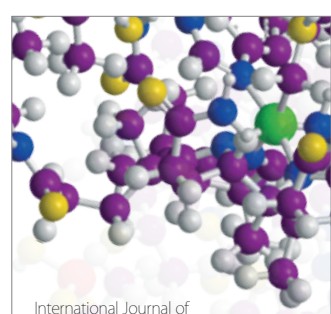

Carbohydrate Chemistry

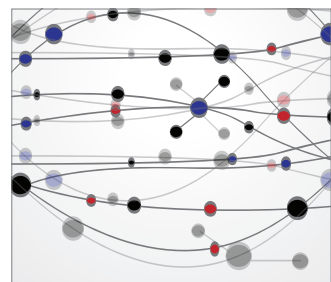

The Scientific World Journal
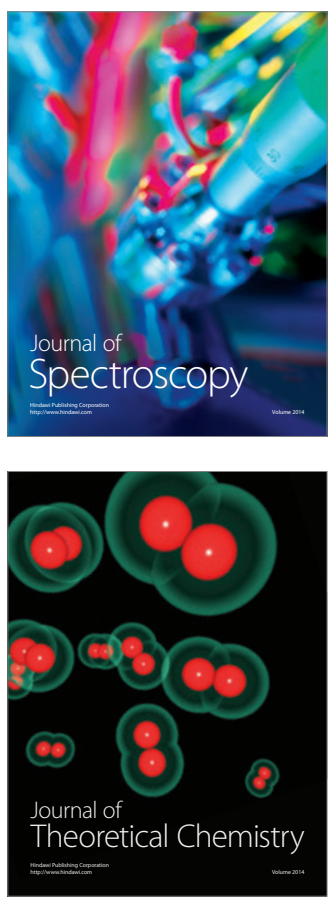
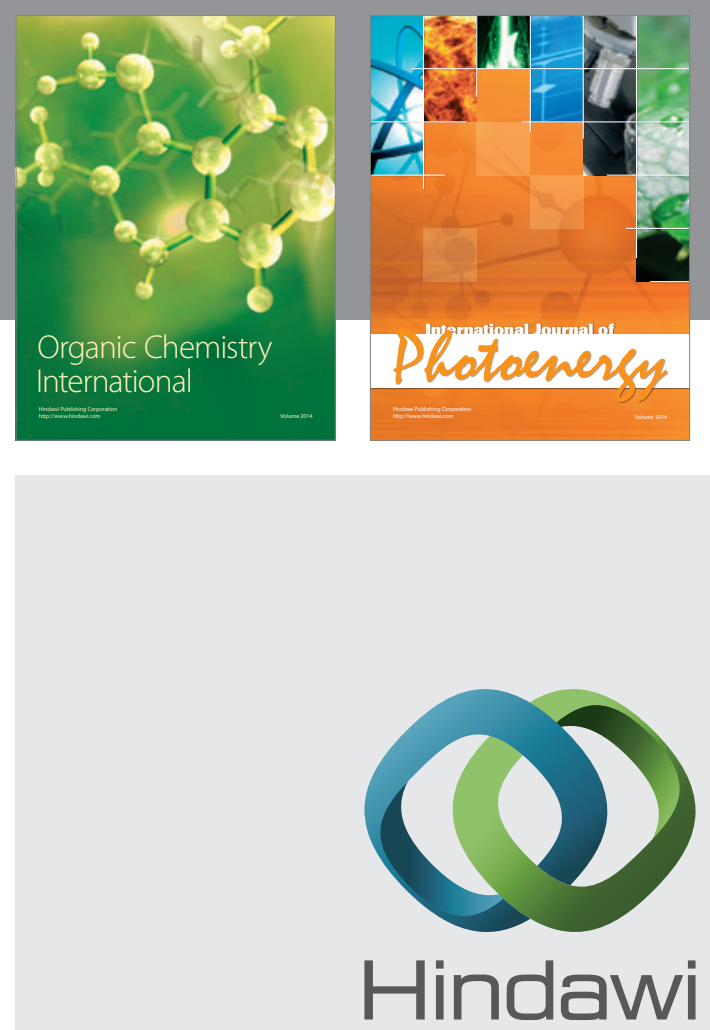

Submit your manuscripts at

http://www.hindawi.com
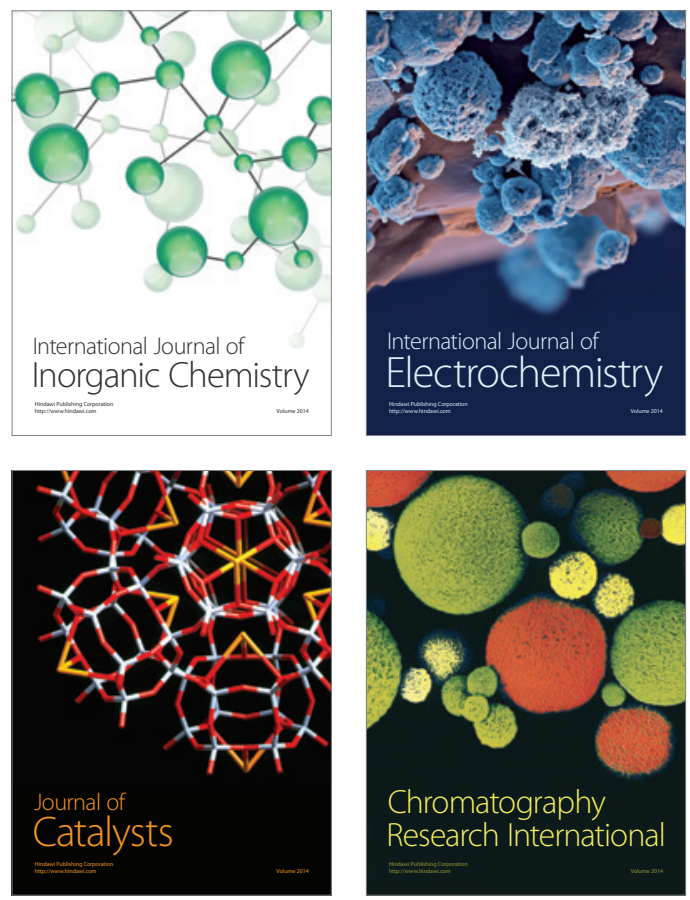
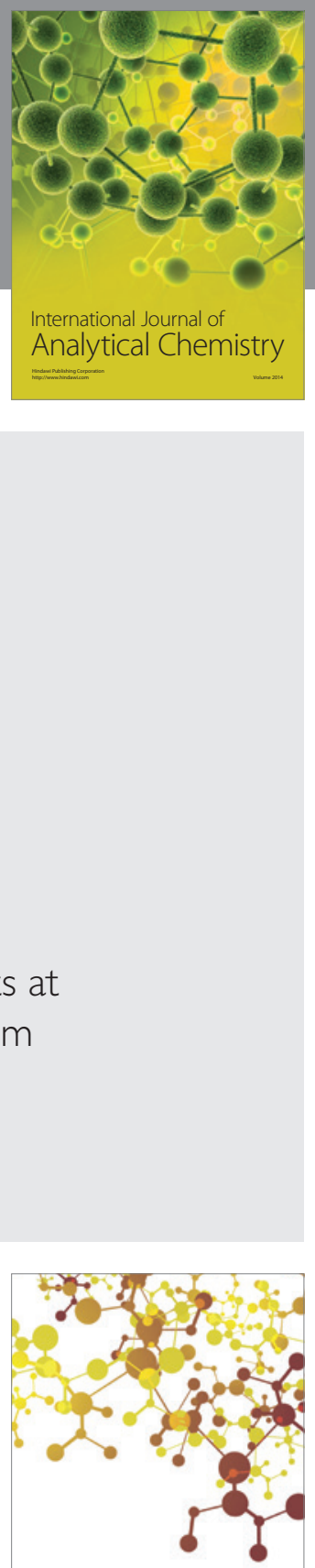

Journal of

Applied Chemistry
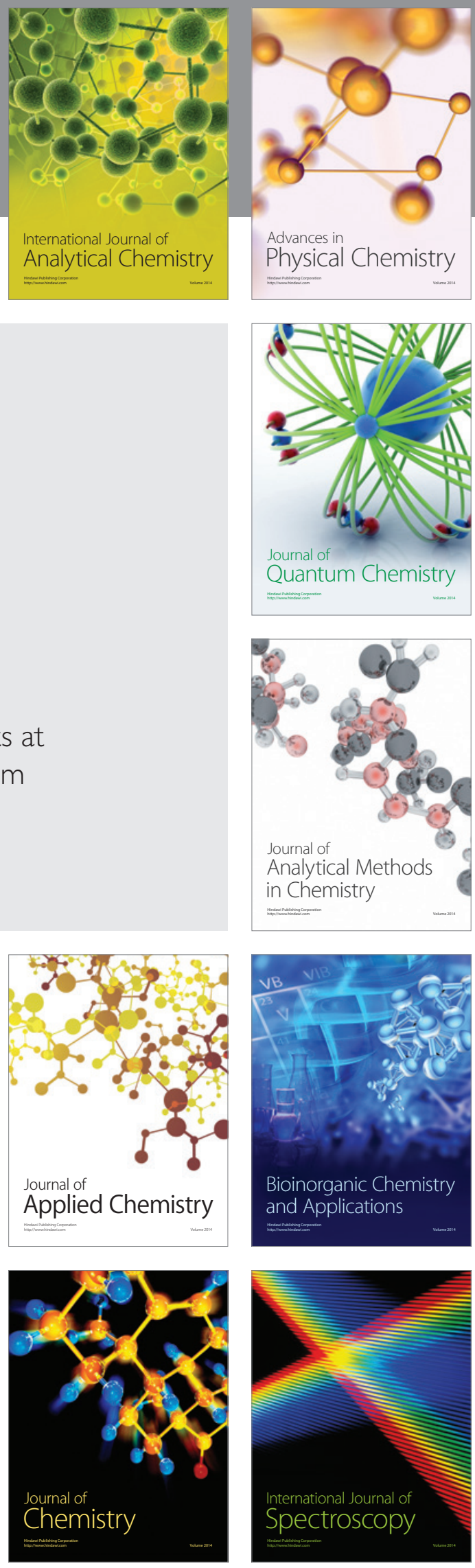